\title{
Alternating Current Electrophoretic Deposition of Multiwall Carbon Nanotubes-Polyaniline for Supercapacitor Electrode
}

\author{
Nor Hamizah Eleas, Nurul Nazwa Mohammad, Azmi Mohamed Yusof, and Intan Syaffinazzilla Zaine
}

\begin{abstract}
The fundamental of electrophoretic deposition (EPD) technique concerns on the use of liquid base colloid to prepare suspension. The pitfall of water based colloid medium has been a tendency for hydrolysis process to occur. Nevertheless, alternating current electrophoretic deposition (AC-EPD) enables water base colloid to be used and at the mean time controls the hydrolysis effect. This study evaluates the effectiveness of deposition thin film Multi Wall Carbon Nanotubes-Polyaniline (MWCNTs-PANi) nanocomposite using AC-EPD. The results were compared with direct current electrophoretic deposition (DC-EPD). FESEM image of AC-EPD shows denser MWCNTs-PANi deposition on nickel substrate. MWCNTs-PANi molecular structures were observed by FTIR analysis. MWCNTs-PANi deposited by AC-EPD consequence to higher specific capacitance and energy density compared to DC-EPD. Specific capacitance of MWCNTs-PANi nanocomposite via AC-EPD and DC-EPD were $22.9 \mathrm{~F} \mathrm{~g}^{-1}$ and $10.8 \mathrm{~F} \mathrm{~g}^{-1}$ respectively. Meanwhile, the energy density of AC-EPD is $0.0023 \mathrm{Wh}^{-1}$ whereas the energy density of DC-EPD is $0.0011 \mathrm{Wh} \mathrm{kg}^{-1}$. This study points out that the AC-EPD has more advantages than DC-EPD technique.
\end{abstract}

Index Terms-Alternating current, carbon nanotubes, electrophoretic deposition, polyaniline.

\section{INTRODUCTION}

Multiwall carbon nanotubes (MWCNTs) conjugated with conducting polymer polyaniline (PANi) have been extensively explored by researchers since this type of nanocomposite material promise such good output in application such as in solar cell [1], biosensor [2] and supercapacitor [3]. Special attention was paid to the supercapacitor application for that study. There are several types of technique to deposit nanocomposite material on substrate [4], [5] yet, electrophoretic deposition (EPD) is one of technique which extensively use due to its easy and versatile method to deposit material on substrate accompany with three fundamental setup requirements which are power supply, deposition electrodes and suspension with charged particles. The most cost effective and environmental friendly way to prepare the suspension is by using water as the liquid

Manuscript received November 7, 2016; revised June 2, 2017. This work was supported in part by the Ministry of Education, Malaysia under Grant Research Acculturation Grant Scheme (RAGS/1/2014/SG06/UITM/6)

Nor Hamizah Eleas, Intan Syaffinazzilla Zaine, and Nurul Nazwa Mohammad are with the Department of Applied Sciences, University Teknologi MARA, Pulau Pinang, Malaysia (e-mail: mizaheleas@gmail.com, intan.zaine@ppinang.uitm.edu.my,nurul.nazwa@ppinang.uitm.edu.my).

Azmi Mohamed Yusof is with the Faculty of Mechanical Engineering, University Teknologi MARA, Pulau Pinang, Malaysia (e-mail: azmi349@ppinang.uitm.edu.my). medium of the charged particles suspension. Unfortunately water may result to hydrolysis which causes poor deposition of material on substrate, as decomposition of water trigger bubbles formation. In EPD technique, direct current (DC) is typically used [6]-[8]. Currently, researchers attention have been diverted into the alternating current electrophoretic deposition (AC-EPD) due to the advantages on ability to minimize water decomposition effect and also enable deposition at high voltage which alleviate efficiency in certain application [9]-[13]. This work evaluates the effectiveness of depositing thin film MWCNTs-PANi on nickel substrate using direct current (DC-EPD) and alternating current (AC-EPD) electrophoretic depositions.

\section{EXPERIMENTAL}

\section{A. Materials and Chemicals}

MWCNTs with a purity of $>95 \%$, outer diameter of $>50$ $\mathrm{nm}$ and length of 10-20 $\mu \mathrm{m}$ was purchased from Universiti Sains Malaysia. All other chemicals that will be used are of analytical reagent grades such as hydrochloric acid with purity of $99.5 \%$ (EMPARTA), polyaniline emaraldine base with purity of $99.5 \%$ (SIGMA ALDRICH) and crystal violet with purity of $90.0 \%$ (MERCK).

\section{B. Methodology}

The electrophoresis performed in the experimental setup consists of DC function generator (PHYWE) or AC generator (GFG-3015) separately. MWCNTs-PANi suspension was prepared by diluting in deionized (DI) water dispersed by crystal violet with two nickel electrodes of thickness $0.1 \mathrm{~mm}$ and purity $>99.5 \%$, one of which is the substrate of films deposition. Nickel foils were cut in dimension of $4.5 \mathrm{~cm} \times 4.5$ $\mathrm{cm}$. Parallel separation distance of the electrodes was $8 \mathrm{~mm}$.

The dispersion of MWCNTs was performed by preparing the suspension through mixing 1g L-1 of MWCNTs in fresh DI water. The suspension was then ultrasonicated for 1 hour before being added with $15 \mathrm{wt} . \%$ crystal violet. Although the liquid based medium need dispersant to support the suspension however, water is preferred in this study since it is cost effective, easy to handle and environmental friendly. The suspensions were then magnetically stirred for 1 hour. The addition of PANi into the suspension was done via ex-situ polymerization. A well-mixed PANi was prepared by mixing polyaniline emaraldine base with $1 \mathrm{M}$ of hydrochloric acid and placed it in orbital shaker for 30 minutes. In order to obtain oxidize PANi. 10 wt.\% of PANi was added into the suspension and was magnetically stirred for another 2 hours followed by 1 hour ultrasonification. 
The electrodes were immersed into colloids and were connected to a programmable function generator (GFG-3015) to generate the asymmetric AC signal while for DC-EPD using separate function generator (PHYWE). The AC signal was a square waveform type and set at frequency of $1 \mathrm{kHz}$ and voltage of $16 \mathrm{Vp}-\mathrm{p}$. The input voltage for DC-EPD was set at $8 \mathrm{~V}$. The deposition times were fixed at 300 seconds for both set-ups. The summary of experimental setting is summarized in Table I. The deposition of thin film is formed on negatively charge electrode during high amplitude section of the signal. The deposited films were dried in the desiccators.

Field emission scanning electron microscope (FESEM) has been used to study the morphology properties of the MWCNTs-PANi films. The films were cut into small pieces and coated with a thin layer of gold using sputter coater that serves as a conductor during the process of image formation. Fourier transform infrared spectroscopy (FTIR) was used to study the structural properties of the films. The electrochemical properties of the films have been studied using cyclic voltammetry (CV) and galvanostatic charge-discharge (CD) measurement. The electrochemical studies of the deposited films are conducted using a commercial three-electrode cell containing $\mathrm{Ag} / \mathrm{AgCl}$ act as reference electrode, platinum plate $(1 \mathrm{~cm} \times 1 \mathrm{~cm})$ as counter electrode and the deposited films as working electrodes. The $6 \mathrm{M} \mathrm{KOH}$ solution was used as electrolyte at scan rate of 5 $\mathrm{mVs}-1$. CV measurements were employed in order to evaluate the specific capacitance of the deposited films. Potential window, $\mathrm{V}$ of $0.45 \mathrm{~V}$ is used for $\mathrm{CV}$ analysis. By using chronopotentiometry technique, 50 cycle-lives with potential window of $0.42 \mathrm{~V}$ was observed.

The specific capacitance, $C_{s}$ of the electrodes can be calculated by equation,

$$
C_{s}=\frac{I m}{S}
$$

where the current $\mathrm{I}$, is the area under the graph obtain from cyclic voltammogram in unit ampere (A), $m$ is the mass of deposited films in unit gram and $S$, is the scan rate in unit $\mathrm{V}$ $\mathrm{s}^{-1}$.

Energy density, $E$ of the electrodes can be calculated using the equation,

$$
E=\frac{1}{2} C_{s} V^{2}
$$

Galvanostatic charge-discharge measurements were conducted using chronopotentiometry at different constant current densities with fixed potential window, $V$ from 0 to $0.75 \mathrm{~V}$. The power density, $P$ for each current density of constant discharging current can be calculated using the following equation,

$$
P=\frac{E}{\Delta t}
$$

where $\Delta t$ is the time of discharging potential range in hours.

All of the variables values were obtain from the cyclic voltammogram and charge discharge curves.
TABLE I: TIMES OF DEPOSITION USED IN THIS STUDY AND THE CORRESPONDING SAMPLE

\begin{tabular}{ccc}
\hline \hline Method & Deposition time (s) & Sample name \\
\hline AC-EPD & 300 & MWCNTs-PANi (AC-EPD) \\
DC-EPD & 300 & MWCNTs-PANi (DC-EPD) \\
\hline \hline
\end{tabular}

\section{RESULTS AND DISCUSSION}

\section{A. Microstructural Properties}
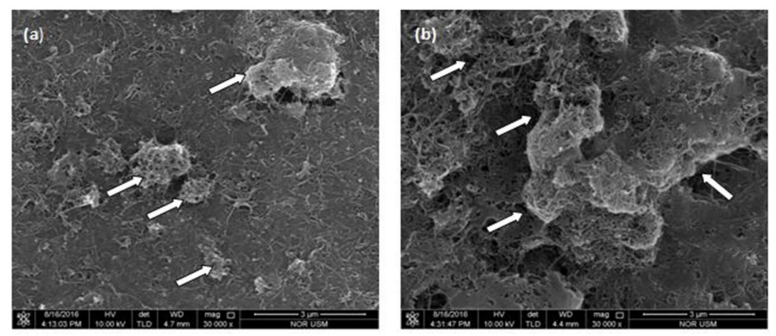

Fig. 1. FESEM image of (a) MWCNTs-PANi (AC-EPD) (b) MWCNTs-PANi (DC-EPD).

Fig. 1(a) and Fig. 1(b) show the FESEM micrograph of the MWCNTs-PANi nanocomposite film by AC-EPD and DC-EPD respectively. Both images were magnified at similar magnification of $30 \mathrm{k}$ shows that the MWCNTs structures been covered by flat sheet structures of PANi. These indicate most of the MWCNTs were coated with a homogenous layer of PANi. MWCNTs-PANi nanocomposite formed a coralloid network on the surface of the films shown by the arrows due to the fact that PANi chains have tendency to bind MWCNTs together in the suspension which affected the film deposition. These results were also agreed with the previous study [14].

Fig. 1(a) shows more uniform and smooth surface with less agglomerated surfaces compared to Fig. 1(b). Fine size and denser MWCNTs-PANi deposition can be observed in Fig. 1(a) compared to Fig. 1(b). The result signifies that many tiny particles of MWCNTs-PANi nanocomposite were successfully deposited on nickel substrate. The morphology images obtained were parallel with previous work which explained that the AC-EPD technique gives better packing of particles that arrange alternately and fill in the deposition area. Meanwhile, the constant voltage in DC-EPD allow the particles to arrange from smaller to bigger size [15]. The surface morphology observes at Fig. 1(b) shows many pore structure compared to Fig. 1(a). This circumstance maybe due to the bubbles formation during DC-EPD that makes MWCNTs-PANi nanocomposite deposited roughly and not uniform.

\section{B. Functional Group Analysis}

Fig. 2(a) and Fig. 2(b) shows the FTIR spectra of MWCNTs-PANi nanocomposite film on Nickel substrate in the region $1000-4000 \mathrm{~cm}^{-1}$ with different type of power supply used, AC and DC respectively. FTIR provide information of functional group exerted on samples. Absorption bands observe at $1156.6 \mathrm{~cm}^{-1}$ and $1212.4 \mathrm{~cm}^{-1}$ while $1162.6 \mathrm{~cm}^{-1}$ and $1356.4 \mathrm{~cm}^{-1}$ in Fig. 2 (a) and Fig. 2 (b) respectively, attributed to the $\mathrm{C}-\mathrm{C}$ stretching predominantly show the characteristics of the MWCNTs as referred to [16]. The absorption band at $1537 \mathrm{~cm}^{-1}$ and $1512 \mathrm{~cm}^{-1}$ for $\mathrm{AC}$ and $\mathrm{DC}$ contributed by $\mathrm{C}=\mathrm{C}$ of the hexagonal network MWCNTs with the agreement with R.Y. Suckeveriene, E. Zelikman, G. 
Mechrez and M. Narkis [17].

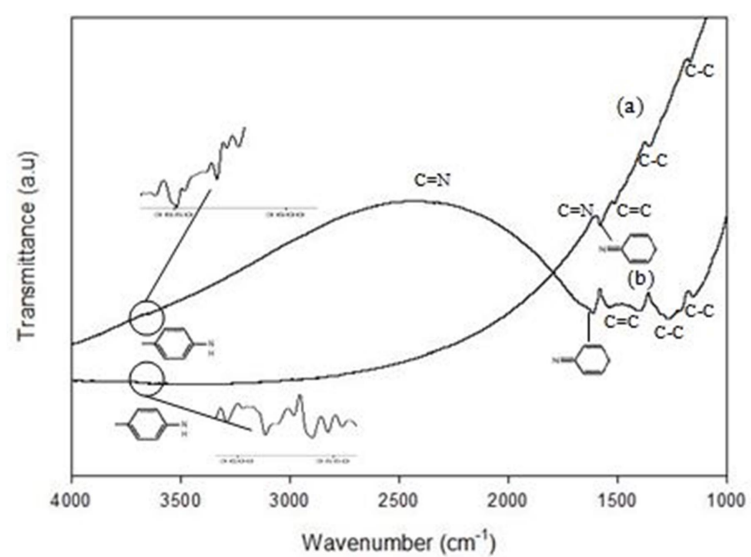

Fig. 2. FTIR spectra (a) MWCNTs-PANi (DC-EPD) (b) MWCNTs PANi (AC-EPD).

Weaker peaks can be observed in Fig. 2(b) compare to Fig. 2(a) were believe due to the poor quality of MWCNTs-PANi deposition via DC-EPD. Meanwhile, the vibrational frequencies observed at $3630 \mathrm{~cm}^{-1}$ for AC-EPD and 3585 $\mathrm{cm}^{-1}$ for DC-EPD shows the N-H stretching frequencies of complexed $\mathrm{NH}_{3}$ and that of PANi with decreased intensity [18]. Hump in MWCNTs-PANi curve by AC-EPD starting at $1612 \mathrm{~cm}^{-1}$ and $1581 \mathrm{~cm}^{-1}$ for DC-EPD shows the dominant play of PANi due to vibrational of the $\mathrm{C}=\mathrm{N}$ and $\mathrm{C}=\mathrm{C}$ stretching vibration from quinoid and benzenoid rings in PANi with C-C bond in MWCNTs as referred to S. Peng, X. Fan, S. Li, and J. Zhang [16], and R. Y. Suckeveriene, E. Zelikman, G. Mechrez, and M. Narkis [17].

The hump observe for MWCNTs-PANi film deposited via AC-EPD were broader that MWCNTs-PANi film deposition by DC-EPD may owed to definite bonding of quinoid and benzenoid with MWCNTs. Meanwhile, the quinoid functional group for both samples at $1612 \mathrm{~cm}^{-1}$ and $1581 \mathrm{~cm}^{-1}$ also can be observed for Fig. 2(a) and Fig. 2(b) respectively with the agreement to the previous work done by S. B. Kondawar, M. D. Deshpande, and S. P. Agrawal [19].

FTIR data shows the deposition of MWCNTs-PANi was successfully obtained via ex-situ polymerization by AC-EPD and DC-EPD technique. On the other hand, MWCNTs-PANi film deposition via AC-EPD film shows stronger band compared to MWCNTs-PANi via DC-EPD. These results also indicate MWCNTs-PANi was efficiently deposited by AC-EPD technique.

\section{Electrochemical Properties}

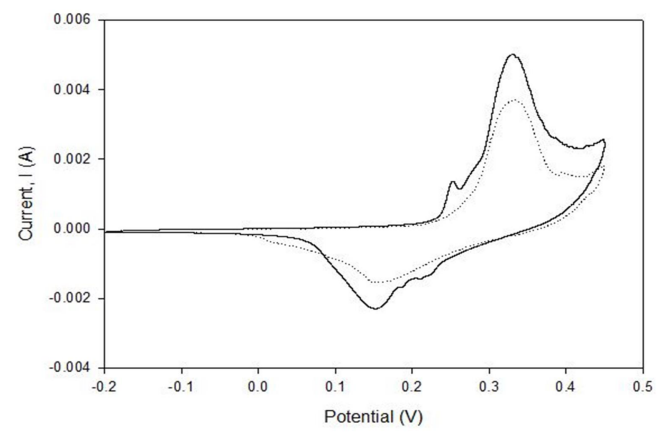

Fig. 3. Cyclic voltammogram of MWCNTs-PANi film deposition via AC-EPD and DC-EPD.

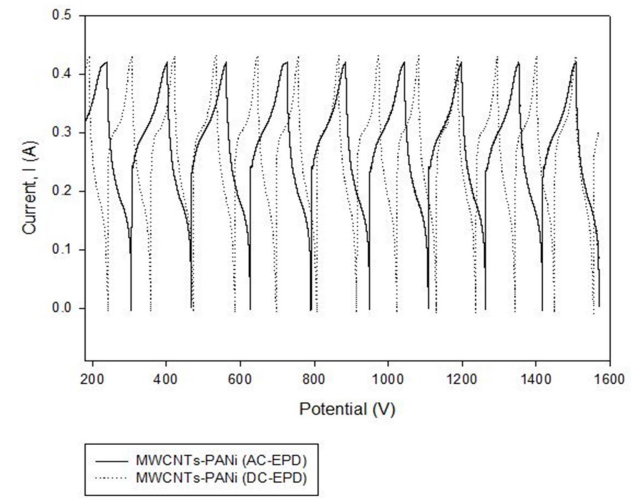

Fig. 4. Charge-Discharge curves of MWCNTs-PANi film deposition via $\mathrm{AC}-\mathrm{EPD}$ and DC-EPD.

The cyclic voltammogram of the electrode for supercapacitor base material MWCNTs-PANi film via AC-EPD and DC-EPD were shown in Fig. 3. The cyclic voltammogram obtain in the MWCNTs-PANi composite electrode for both AC and DC field EPD strongly demonstrated a 'psuedocapacitance' effect, whereby charge was stored and released through faradaic reaction of oxidation and reduction of MWCNTs-PANi on the surface of the electrode. The two prominent peak of anodic and chatodic scan for MWCNTs-PANi deposition via AC-EPD was higher than DC-EPD clearly imply the oxidation process occur and yielded higher current with the agreement to C. Y. Chen, Z. Y. Shih, Z. Yang, and H. T. Chang [20].

Higher total area in cyclic voltammogram of AC-EPD MWCNTs-PANi sample point towards higher specific capacitance compared to MWCNTs-PANi DC-EPD. For MWCNTs-PANi via AC-EPD curves, there were another peaks existed during anodic and cathodic scan which define there were two oxidation and reduction peaks for AC-EPD compare to DC-EPD whereas only one pair of oxidation and reduction peaks can be observed. This result also reveals PANi structure in MWCNTs by AC-EPD was definite as observed in FTIR result in Fig. 2.

From cyclic voltammogram, the specific capacitance and energy density were calculated for both samples which conclude the value of specific capacitance of MWCNTs-PANi nanocomposite via AC-EPD and DC-EPD were $22.9 \mathrm{~F} \mathrm{~g}^{-1}$ and $10.8 \mathrm{~F} \mathrm{~g}^{-1}$ respectively. Meanwhile, the energy density of AC-EPD is $0.0023 \mathrm{Wh} \mathrm{kg}^{-1}$ whereby the energy density of DC-EPD is $0.0011 \mathrm{Wh} \mathrm{kg}^{-1}$. Meanwhile, the power density were calculated from the discharge time obtain from charge discharge curves shown in Fig. 4. Power density calculated was $0.12 \mathrm{~W} \mathrm{~kg}^{-1}$ for MWCNTs-PANi (AC-EPD) and $0.08 \mathrm{~W} \mathrm{~kg}^{-1}$ for MWCNTs-PANi (DC-EPD). All values calculated were shown in Table II.

TABLE II: SPECIFIC CAPACITANCE, ENERGY DENSITY AND POWER DENSITY FOR MWCNTS-PANI (AC-EPD) AND MWCNTS-PANI (DC-EPD)

\begin{tabular}{ccc} 
Properties & $\begin{array}{c}\text { MWCNTs-PANi } \\
\text { (AC-EPD) }\end{array}$ & $\begin{array}{c}\text { MWCNTs-PANi } \\
\text { (DC-EPD) }\end{array}$ \\
\hline $\begin{array}{c}\text { Specific capacitance } \\
\left(\mathrm{F} \mathrm{g}^{-1}\right)\end{array}$ & 22.9 & 10.8 \\
$\begin{array}{c}\text { Energy density (Wh } \\
\left.\mathrm{kg}^{-1}\right)\end{array}$ & 0.0023 & 0.0011 \\
$\begin{array}{c}\text { Power density (W } \\
\left.\mathrm{kg}^{-1}\right)\end{array}$ & 0.12 & 0.08 \\
\hline \hline
\end{tabular}

These results show AC-EPD technique not only improves 
the deposition quality, in fact also makes MWCNTs-PANi were well deposited and responsible for good electrochemical response. The homogenous surface of MWCNTs-PANi (AC-EPD) deposited on substrate observed by FESEM in Fig. 1 also shows the ability of electrolyte access efficiently during the redox activity takes place.

The non linear charge discharge curve from Fig. 4 indicates MWCNTs-PANi film deposited for both samples of produce redox activity during charge discharge process. The charge discharge counter parts are stable until 50 cycles of charge discharge process.

\section{CONCLUSION}

These studies compare electrophoretic deposition carried out under AC and DC electric field conditions. The results showed that the AC-EPD method leads to improved quality of deposited MWCNTs-PANi as compared to DC-EPD. Subsequently, the improved quality of MWCNTs-PANi deposition by AC-EPD also improves the specific capacitance and its power density in electrochemical supercapacitor application.

\section{ACKNOWLEDGMENT}

The authors would like to thank the Ministry of Education, Malaysia that supports this work through Research Acculturation Grant Scheme (RAGS/1/2014/SG06/UITM/6).

\section{REFERENCES}

[1] Y. Xiao, J. Y. Lin, J. Wu, S. Y. Tai, G. Yue, and T. W. Lin, "Dye-sensitized solar cells with high-performance polyaniline/multi-wall carbon nanotube counter electrodes electropolymerized by a pulse potentiostatic technique," J. Power Sources, vol. 233, pp. 320-325, 2013.

[2] E. Bayram and E. Akyilmaz, "Development of a new microbial biosensor based on conductive polymer/multiwalled carbon nanotube and its application to paracetamol determination," Sensors Actuators B. Chem., 2016.

[3] N. Hui, F. Chai, P. Lin, Z. Song, X. Sun, Y. Li, S. Niu, and X. Luo, "Electrodeposited conducting polyaniline nanowire arrays aligned on carbon nanotubes network for high performance supercapacitors and sensors," Electrochim. Acta, vol. 199, pp. 234-241, 2016.

[4] L. Delzeit, C. V. Nguyen, B. Chen, R. Stevens, A. Cassell, J. Han, and M. Meyyappan, "Multiwalled carbon nanotubes by chemical vapor deposition using multilayered metal catalysts," J. Phys. Chem. B, vol. 106, pp. 5629-5635, 2002.

[5] M. Sánchez and M. E. Rincón, "Sensor response of sol-gel multiwalled carbon nanotubes-TiO2 composites deposited by screen-printing and dip-coating techniques," Sensors Actuators, B Chem., vol. 140, no. 1, pp. $17-23,2009$.

[6] A. R. Boccaccini, J. Cho, J. A. Roether, B. J. C. Thomas, E. Jane Minay, and M. S. P. Shaffer, "Electrophoretic deposition of carbon nanotubes," Carbon N. Y., vol. 44, no. 15, pp. 3149-3160, 2006.

[7] A. Sarkar and T. Daniels-Race, "Electrophoretic deposition of carbon nanotubes on 3-Amino-Propyl-Triethoxysilane (APTES) surface functionalized silicon substrates," Nanomaterials, vol. 3, no. 2, pp. 272-288, 2013.

[8] M. Diba, D. W. H. Fam, A. R. Boccaccini, and M. S. P. Shaffer, "Electrophoretic deposition of graphene-related materials: A review of the fundamentals," Prog. Mater. Sci., vol. 82, pp. 83-117, 2016.

[9] B. Neirinck, F. Singer, A. Braem, S. Virtanen, and J. Vleugels, "Alternating Current Electrophoretic Deposition of Bovine Serum Albumin onto Magnesium," Key Eng. Mater., vol. 654, pp. 139-143, 2015.

[10] M. Ammam, "Electrophoretic deposition under modulated electric fields: a review," RSC $A d v$., vol. 2, no. 20, p. 7633, 2012.
[11] D. H. Yoon and K. Raju, "Alternating current electrophoretic deposition ( ac-epd ) of sic nanoparticles in an aqueous suspension for the fabrication of sic f / sic composites," Digest Journal of Nanomaterials, vol. 10, no. 3, pp. 1103-1108, 2015.

[12] M. Navidirad, B. Raissi, R. Riahifar, M. S. Yaghmaee, and A. Kazemzadeh, "Effect of polyethylenimine on electrophoretic deposition of TiO2 nanoparticles in alternating current electric field," $J$. Mater. Sci. Mater. Electron., vol. 25, no. 11, pp. 5041-5050, 2014.

[13] S. Seuss, M. Lehmann, and A. R. Boccaccini, "Alternating current electrophoretic deposition of antibacterial bioactive Glass-Chitosan composite coatings," Int. J. Mol. Sci., vol. 15, no. 7, pp. 12231-12242, 2014.

[14] C. Peng, S. Zhang, D. Jewell, and G. Z. Chen, "Carbon nanotube and conducting polymer composites for supercapacitors," Prog. Nat. Sci., vol. 18, no. 7, pp. 777-788, 2008.

[15] V. Ozhukil Kollath, Q. Chen, R. Closset, J. Luyten, K. Traina, S. Mullens, A. R. Boccaccini, and R. Cloots, "AC vs. DC electrophoretic deposition of hydroxyapatite on titanium," J. Eur. Ceram. Soc., vol. 33, no. 13-14, pp. 2715-2721, 2013.

[16] S. Peng, X. Fan, S. Li, and J. Zhang, "Green synthesis and characterization of graphite oxide by orthogonal experiment," J. Chil. Chem. Soc., vol. 58, no. 4, pp. 2213-2217, 2013.

[17] R. Y. Suckeveriene, E. Zelikman, G. Mechrez, and M. Narkis, "Literature review: Conducting carbon nanotube/polyaniline nanocomposites," Rev. Chem. Eng., vol. 27, no. 1-2, pp. 15-21, 2011.

[18] W. Naqash and K. Majid, "Synthesis, characterization and study of effect of irradiation on electronic properties of polyaniline composite with metal complex of Co ( III )," vol. 18, no. 5, pp. 1121-1127, 2015

[19] S. B. Kondawar, M. D. Deshpande, and S. P. Agrawal, "Transport properties of conductive polyaniline nanocomposites based on carbon nanotubes," Int. J. Compos. Mater., vol. 2, no. 3, pp. 32-36, 2012.

[20] C. Y. Chen, Z. Y. Shih, Z. Yang, and H. T. Chang, "Carbon nanotubes/cobalt sulfide composites as potential high-rate and high-efficiency supercapacitors," J. Power Sources, vol. 215, pp. 43-47, 2012.

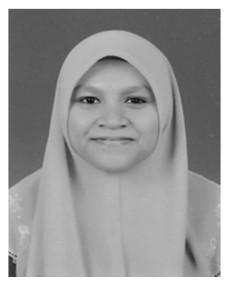

Nor Hamizah Eleas was born in 1988 in Perak, Malaysia. She graduated from Universiti Teknologi MARA (UiTM) Shah Alam, Malaysia in Bachelor of Science Physics. She then join Electrophoretic Deposition Research group at UiTM Pulau Pinang branch from Disember 2015 until now. Currently she is a master candidate in science and material technology, UiTM Pulau Pinang, Malaysia.

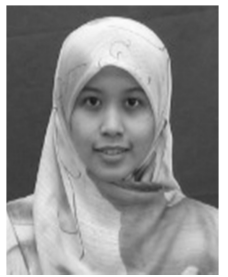

Nurul Nazwa Mohammad was born in 1986 in Sarawak, Malaysia. She graduated from University Sains Malaysia (USM) in Master of Solid State Physics in 2010. Currently she is working as a lecturer at Applied Science Department, Universiti Teknologi MARA (UiTM ) Pulau Pinang Malaysia.

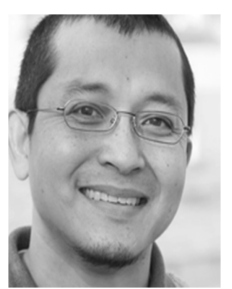

Azmi Mohamed Yusof received the Ph.D. degree in Microsystem Engineering from University of Freiburg, Germany in 2012. He has been with Electrophoretic Deposition Research group at Universiti Teknologi MARA (UiTM) Pulau Pinang, Malaysia since 2013. Currently he is a senior faculty member at UiTM Pulau Pinang, Malaysia.

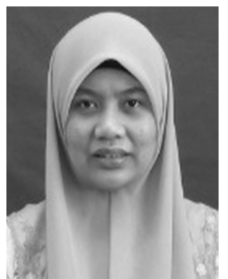

Intan Syaffinazzilla Zaine graduated from Universiti Kebangsaan Malaysia (UKM), Bangi, Malaysia in Master of Science (Applied Physics). She then was appointed as lecturer (Physics) at Universiti Teknologi MARA (UiTM) Pulau Pinang, Malaysia in 2008 before founded Electrophoretic Deposition Research group in 2014. Currently she is a senior lecturer (Physics) at UiTM Pulau Pinang, Malaysia. 